\title{
Distinction between distribution of masses of Wolf-Rayet stars and that of relativistic objects
}

\author{
Anatol M. Cherepashchuk \\ Sternberg Astronomical Institute, \\ 13, Universitetskii Prospekt, Moscow, 119992, Russia
}

\begin{abstract}
The final masses $M_{\mathrm{CO}, \mathrm{f}}$ for the CO-cores of WR stars with known masses are calculated taking into account mass-dependent mass loss of WR stars and clumping structure of the WR wind which allows the mass loss rate to be decreased by a factor of 3 . The masses of $M_{\mathrm{CO}, \mathrm{f}}$ lie in the range (1-2)$(20-44) \mathrm{M}_{\odot}$ and have continuous distribution in contrast with distribution of masses $M_{\mathrm{x}}$ of relativistic objects. The distribution of $M_{\mathrm{x}}$ seems to be bimodal with a gap in the range $M_{\mathrm{x}}=2-4 \mathrm{M}_{\odot}$. A mean CO-core mass $\left\langle M_{\mathrm{CO}, \mathrm{f}}\right\rangle=7.4-$ $10.3 \mathrm{M}_{\odot}$ is close to that of black holes: $\left\langle M_{\mathrm{BH}}\right\rangle=8-10 \mathrm{M}_{\odot}$. Difference between distributions of $M_{\mathrm{CO}, \mathrm{f}}$ and $M_{\mathrm{x}}$ allows us to suggest that the nature of a formed relativistic object (neutron star, black hole) is determined not only by the mass of a progenitor but also by some other parameters: rotation, magnetic field, etc.
\end{abstract}

\section{Introduction}

WR stars, being as they are massive, basically helium stars at a late evolutionary stage; should explode as supernovae of Types Ib or Ic and form relativistic objects as a result of collapse of their CO-cores. Because WR stars have no massive hydrogen enyelopes, it makes easier for them to transform the collapse energy into the observed gamma-ray radiation. Therefore, collapses of CO-cores of WR stars may produce $\gamma$-ray bursts (Gershtein 2000; Postnov \& Cherepashchuk 2001). Up to now the masses of $23 \mathrm{WR}$ stars in WR+O binary systems have been measured and those of 34 relativistic objects (19 neutron stars and 15 black holes) in X-ray binary systems and binary systems with radiopulsars have been estimated (van der Hucht 2001; Cherepashchuk 2001; Charles 2001; Thorsett \& Chakrabarty 1998). It is interesting to compare the masses of WR stars and their CO-cores at the end of their evolution with the corresponding parameters of relativistic objects.

Not only WR stars can produce relativistic objects but also blue and red supergiants. Comparison of the masses of WR stars and relativistic objects, however, seems to be correct because we consider black holes and neutron stars in binary systems: massive O-B stars in binary systems lose their hydrogen envelopes due to mass exchange and form WR stars. Most evolutionary scenaria for close binaries containing massive O-B stars predict formation of a WR star with the core collapse leading to the formation of a relativistic object (e.g., Shore et al. 1994; Iben et al. 1995). Only a few radiopulsars in binary systems with circular orbits and low-mass white dwarfs as companions may be formed not through WR stars but as a result of collapse of white dwarfs after having 
increased their masses due to accretion of matter up to Chandrasekhar's limit (Shore et al. 1994). Therefore, there is strong evidence to suggest that all relativistic objects in X-ray binaries and most pulsars in binary systems have been formed as a result of collapse of CO-cores of WR stars.

\section{Distribution of masses of relativistic objects}

As was pointed out by Bailyn et al. (1998) and Cherepashchuk (1998), the distribution of masses of relativistic objects is bimodal: masses of neutron stars lie in the small range, $m_{\mathrm{NS}}=1-2 \mathrm{M}_{\odot}$, masses of black holes are distributed in the range $4-16 \mathrm{M}_{\odot}$ (Charles 2001; Thorsett \& Chakrabarty 1998; Cherepashchuk 2001). The neutron star in X-ray binary Vela X-1 is known to have the greatest mass among objects of the kind, $M_{\mathrm{NS}}=1.86 \pm 0.16 \mathrm{M}_{\odot}$ (Barziv et al. 2001), which does not exceed $2 \mathrm{M}_{\odot}$. In the range of the masses of relativistic objects, $2-4 \mathrm{M}_{\odot}$, is a gap. The average (19 objects) neutron star mass is $\left\langle M_{\mathrm{NS}}\right\rangle=1.35 \pm 0.15 \mathrm{M}_{\odot}$, the average (15 objects) black hole mass is $<M_{\mathrm{BH}}>=8-10 \mathrm{M}_{\odot}$.

As was stressed by Bailyn et al. (1998) and Cherepashchuk (2000, 2001) the gap in the range $2-4 \mathrm{M}_{\odot}$ in the mass distribution for relativistic objects can not be due to observational selection effects.

\section{Distribution of masses of WR stars and their CO-cores at the end of evolution}

To compare masses of WR stars with those of relativistic objects we must take into account mass-dependent mass loss by WR stars (Langer 1989) and clumping structure of WR wind (Cherepashchuk 1990). Because of clumping, the mass loss rate derived from radio and IR data can be decreased by a factor of $\sim 3$ (e.g., Nugis et al. 1998).

Using the approximation formula for $\dot{M}_{\text {WR }}$ obtained from the analysis of polarimetric observations of dozen WR+O stars (Moffat 1995)

$$
\dot{M}_{\mathrm{WR}} \sim k M_{\mathrm{WR}}^{\alpha} \quad \alpha=1-2
$$

and taking the value of coefficient $k$ three times less than applied in previous works (e.g., Langer 1989) we obtained a solution of differential equation (1):

$$
\begin{array}{cc}
M_{\mathrm{WR}}(t)=M_{\mathrm{WR}}^{i} \exp \left(-7 \cdot 10^{7} t\right) & \alpha=1 \\
M_{\mathrm{WR}}(t)=\frac{M_{\mathrm{WR}}^{i}}{1+7 \cdot 10^{-8} M_{\mathrm{WR}}^{i} t} & \alpha=2
\end{array}
$$

In these formulae time $t$ is to be taken in years and the initial mass of a WR star, $M_{\mathrm{WR}}^{i}$, is expressed in solar units. Using the formula for the life-time of a WR star

$$
T=1.74 \cdot 10^{6}\left(\frac{M}{\mathrm{M}_{\odot}}\right)^{-1 / 2}
$$

and that for the mass of a CO-core (Paczynski 1971)

$$
\frac{M_{\mathrm{CO}}}{\mathrm{M}_{\odot}} \simeq 0.45\left(\frac{M_{\mathrm{He}}}{\mathrm{M}_{\odot}}\right)^{1.2}
$$




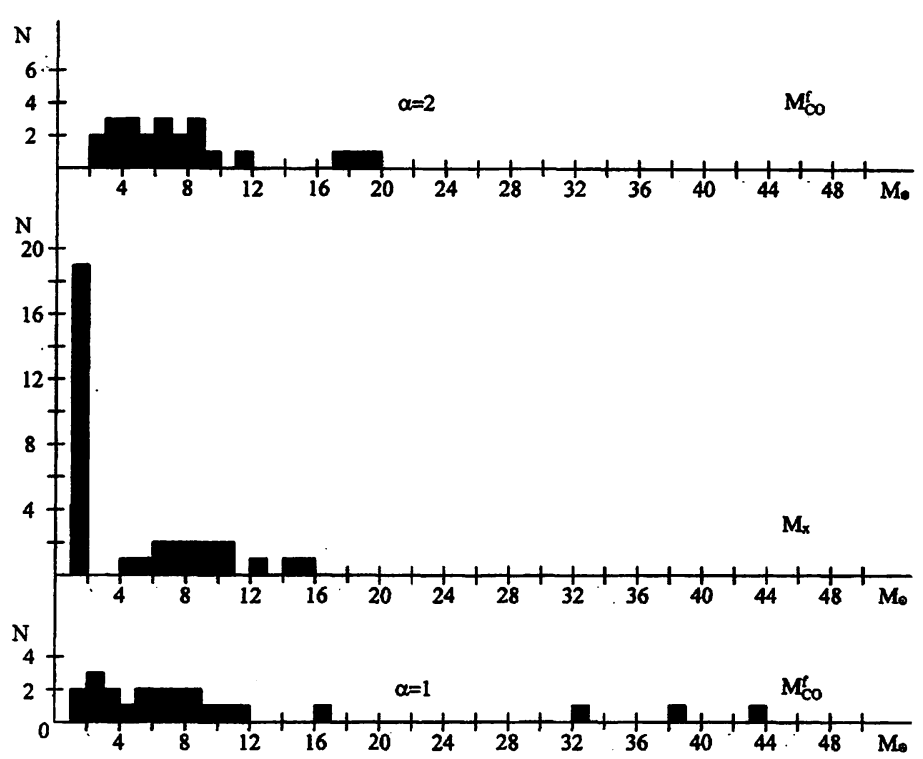

Figure 1. Distributions of $M_{\mathrm{CO}, \mathrm{f}}$ of WR stars with known masses ( $u p: \alpha=2$; down: $\alpha=1$ ). Distribution of masses $M_{x}$ of relativistic objects is presented in the middle of the figure. The peak distribution in the range $M_{\mathrm{x}}=1-2 \mathrm{M}_{\odot}$ is for neutron stars. Distributions of $M_{\mathrm{CO}, \mathrm{f}}$ are continuous although that of $M_{\mathbf{x}}$ is bimodal with the gap in the $M_{\mathrm{x}}=2-4 \mathrm{M}_{\odot}$ range.

it is easy to calculate final masses of CO-cores of WR stars with known masses if an iteration procedure is applied (Cherepashchuk 2001). Values of $M_{\mathrm{CO}, \mathrm{f}}$ calculated in such a way are to be considered as lower limits because real ages of observed-WR stars are not known. Results obtained in such calculations are presented in Figure 1 where the distribution of masses over 34 relativistic objects is given together with that of final masses of CO-cores, $M_{\mathrm{CO}, \mathrm{f}}$, for $23 \mathrm{WR}$ stars with known masses (values of the parameter $\alpha=1$ and 2, correspondingly).

In contrast to relativistic objects, distribution of $M_{\mathrm{CO}, \mathrm{f}}$ is seen to be continuous rather than bimodal in all cases. The gaps in the distributions of $M_{\mathrm{CO}, \mathrm{f}}$ for high masses $M_{\mathrm{CO}, \mathrm{f}}>12 \mathrm{M}_{\odot}$ are due to poor statistics for high-mass WR stars. The distribution of $M_{\mathrm{CO}, \mathrm{f}}$ is wide, $M_{\mathrm{CO}, \mathrm{f}}=(1-2)-(20-44) \mathrm{M}_{\odot}$, and embraces the range of masses of relativistic objects, $M_{\mathrm{x}}=1-16 \mathrm{M}_{\odot}$. Thus, the well known convergence effect for masses of CO-cores of WR stars (Langer 1989) is not realized in our case. It is due to the smaller values of $\dot{M}_{\text {WR }}$ and the smaller power parameter $\alpha=1$ - 2 compared with those traditionally used $(\alpha \simeq 2.5)$. The mean mass of a CO-core is $\left\langle M_{\mathrm{CO}, \mathrm{f}}\right\rangle=7.7 \mathrm{M}_{\odot}$ for $\alpha=2$ and $\left\langle M_{\mathrm{CO}, \mathrm{f}}\right\rangle=10.6 \mathrm{M}_{\odot}$ for $\alpha=1$. The mean value of CO-core $\left\langle M_{\mathrm{CO}, \mathrm{f}}\right\rangle=(7.7-10.6) \mathrm{M}_{\odot}$ is close to the mean mass of black holes, $M_{\mathrm{BH}}=(8-10) \mathrm{M}_{\odot}$. Therefore, the mass of a Type $\mathrm{Ib} / \mathrm{c}$ supernova ejecta during CO-core collapse and formation of a relativistic object is suggested to be rather small.

Recently, maximum supernova luminosities, estimated over 18 well investigated Type Ib/c SNe, have been suspected to have a bi-modal distribution (Richardson et al. 2002). The mean absolute $B$-magnitude at maximum is 
$\mathrm{M}_{B}=-17.1 \mathrm{mag}$ for normal Type $\mathrm{Ib} / \mathrm{c}$ supernovae $(13 \mathrm{SNe})$ and $\mathrm{M}_{B}=-20.26 \mathrm{mag}$ for bright Type Ib/c supernovae ( $5 \mathrm{SNe}$ ). Although insufficient as they are, there are statistical data which indicate that maximum luminosities of normal and bright Type Ib/c supernovae differ from each other by an order of magnitude.

\section{Conclusions}

The distribution of masses $M_{\mathrm{x}}$ observed for relativistic objects is bi-modal. The distribution of maximum luminosities of Type $\mathrm{Ib} / \mathrm{c}$ supernovae may be suggested to be bi-modal too. But the final masses of CO-cores of WR stars (progenitors of relativistic objects) are found to be distributed continuously: $M_{\mathrm{CO}, \mathrm{f}}=(1-2)$ $(20-44) \mathrm{M}_{\odot}$. Such a strong distinction between distributions of $M_{\mathbf{x}}$ and $M_{\mathrm{CO}, \mathrm{f}}$ allows us to suggest that the nature of relativistic objects (neutron stars, black holes) formed during the core collapse of massive stars is determined not only by their mass but also by other parameters, like rotation, magnetic field, random outcome of the core collapse due to some instabilities, etc., (e.g., Tutukov \& Cherepashchuk 1985; Ergma \& van den Heuvel 1998). Rotational effects for some WR stars have been measured recently from de-polarization of emission lines radiation (e.g., Harries et al. 1998). The fraction of rapidly rotating WR stars is $\sim 15-20 \%$. Because of the aspects considered, further investigation of mass distribution among WR stars and relativistic objects seems to be very interesting. Theoretical investigations in this field are also of great interest (e.g., Ivanova \& Chechetkin 1981; Ensman \& Woosley 1988).

According to Postnov \& Prokhorov (2001), the gap in the mass distribution for relativistic objects can be explained in terms of a soft equation of state for the matter of neutron stars (upper limit for the neutron star mass is $1.5 \mathrm{M}_{\odot}$ ) as well as a magneto-rotational mechanism involved (Bisnovatyi-Kogan 1970). In this case the process of matter falling back from supernova envelope onto a magnetized rapidly rotating neutron star may be suppressed for some values of neutron star parameters. There is another possibility to explain the gap, which suggests that the dependence of supernova explosion energy on the progenitor mass is a kind of step-function (Fryer \& Kalogera 2001): $E_{\exp }=2.5 \cdot 10^{51} \mathrm{ergs}^{-1}$ for $M_{\text {prog }}<23 \mathrm{M}_{\odot}$ and $E_{\exp }=0$ for $M_{\text {prog }} \geq 23 \mathrm{M}_{\odot}$.

\section{References}

Bailyn, C.D., Jain, R.K., Coppi P., Orosz, J.A. 1998, ApJ 499, 367

Barziv, O., Kaper, L., van Kerkwijk, M.H., et al. 2001, A\&A 377, 925

Bisnovatyi-Kogan, G.S. 1970, Astron. Zh. 47, 813 (= 1971, Soviet Astron. 14, 652)

Charles, P. 2001, in: L. Kaper, E.P.J. van den Heuvel \& P.A. Woudt (eds.), Black Holes in Binaries and Galactic Nuclei, ESO Astrophysics Symposia (Berlin: Springer), p. 27

Cherepashchuk, A.M. 1990, Astron. Zh. 67, 955 (= Soviet Astron. 34, 481)

Cherepashchuk, A.M. 1998, in: D.S. Wiebe (ed.), Modern Problems of Stellar Evolution, Proc. Int. Conf. Zvenigorod (Moscow: Geos Edition), p. 198

Cherepashchuk, A.M. 2000, Space Sci. Reviews 93, 473

Cherepashchuk, A.M. 2001, Astron. Zh. 78, 145 (= Astron. Reports 45, 120)

Ensman, L.M., Woosley, S.E. 1988, ApJ 333, 754

Ergma, E., van den Heuvel, E.P.J. 1998, A\&A (Letters) 331, L29 
Fryer, C.L., Kalogera, V. 2001, ApJ 554, 548

Gershtein, S.S. 2000, Pis'ma Astron. Zh. 26, 848 (= Astron. Letters 26, 730)

Harries, T.J., Hillier, D.J., Howarth, I.D. 1998, MNRAS 296, 1072

van der Hucht, K.A. 2001, New Astron. Reviews 45, 135

Iben, I., Tutukov, A.V., Yungelson, L.R. 1995, ApJS 100, 233

Ivanova, L.N., Chechetkin, V.M. 1981, Astron. Zh. 58, 1028 (= Soviet Astron. 25, 548)

Langer, N. 1989, A\&A 220, 135

Moffat, A.F.J. 1995, in: K.A. van der Hucht \& P.M. Williams (eds), Wolf-Rayet Stars: Binaries, Colliding Winds, Evolution, Proc. IAU Symp. No. 163 (Dordrecht: Kluwer), p. 213

Nugis, T., Crowther, P.A., Willis, A.J. 1998, A\&A 333, 956

Paczynski, B. 1971, Acta Astron. 21, 1

Postnov, K.A., Cherepashchuk, A.M. 2001, Astron. Zh. 78, 602 (= Astron. Reports 45, 517)

Postnov, K.A., Prokhorov, M.E. 2001, Astron. Zh. 78, 1025 (= Astron. Reports 45, 899)

Richardson, D., Branch, D., Casebeer, D., et al. 2002, AJ 123, 745

Shore, S.N., Livio, M., van den Heuvel, E.P.J. 1994, in: H. Nussbaumer \& A. Orr (eds.), Interacting Binaries, Lecture Notes of the 22nd Advanced Saas-Fee Cource of the SSAA (Berlin: Springer)

Thorsett, S.E., Chakrabarty, D. 1998, ApJ 512, 288

Tutukov, A.V., Cherepashchuk, A.M. 1985, Astron. Zh. 62, 1124 (= Soviet Astron. 29, 654)

\section{Discussion}

LANGER: I find it difficult to compare the WR+O binary sample with the BH binaries: The low mass $\mathrm{BH}$ binaries have low- and intermediate-mass donor stars, i.e., their progenitor stage was not WR+0, and there is practically just one high mass BH binary in our Galaxy: 'Cyg X-1, of which we showed (Brown et al. 2001, New Astron. 6, 457) that it evolved through Case $\mathrm{C}$ mass transfer.

CherepashuK: I compare the masses of WR stars, determined from investigations of $\mathrm{WR}+\mathrm{O}$ binaries, with those of relativistic objects (neutron stars and black holes) in close binary systems. It is widely believed that in the most of the cases progenitors of relativistic objects in close binary systems must be WR stars, but not red and blue supergiants. The problem of evolutionary links between close binary systems of various types containing WR stars and relativistic objects is a particular one. This important problem is not a subject of my talk.

WALBORN: A purely morphological observation that may or may not have any physical relevance is that the mass gap for collapsed objects is reminiscent of the fact that stars up to $8 \mathrm{M}_{\odot}$ can lose enough mass to reach the white-dwarf limit. If $\mathrm{CO}$ cores up to $4 \mathrm{M}_{\odot}$ should lose enough mass during the SN event to reach the neutron-star limit, then the observed effect would be explained.

CherepashchuK: We calculated final masses of CO cores before the collapse. Lifetimes of these $\mathrm{CO}$ cores, progenitors of SNe and relativistic objects, are too short (less than $1000 \mathrm{yr}$ ) and radial mass loss through stellar wind for them is rather small. Estimation of the value of mass loss during the $\mathrm{SNe}$ is a special problem for CO-cores, which could be solved after detailed calculation of the physics of CO-core collapse. 